\title{
Large-scale optimal VAR planning by hybrid simulated annealing/genetic algorithm
}

\author{
Wu-Shun Jwo ${ }^{\mathrm{a}}$, Chih-Wen Liu ${ }^{\mathrm{b}, *}$, Chun-Chang Liu ${ }^{\mathrm{b}}$ \\ ${ }^{a}$ Department of Electrical Engineering, Chien-Kuo Junior College of Technology and Commerce, Changhwa, Taiwan, ROC \\ ${ }^{\mathrm{b}}$ Room 243, Department of Electrical Engineering, National Taiwan University, Taipei, Taiwan, ROC
}

\begin{abstract}
This paper presents a fast near-global optimization technique called hybrid simulated annealing/genetic algorithm (HSAGA) for solving optimal VAR planning problems. The HSAGA incorporates simulated annealing into genetic algorithms to improve both performances at the same time. Therefore, it has the ability to find the near-global optimal solution in a finite time. Moreover, the solution time is much less than that of the conventional simulated annealing method. The HSAGA was applied to a practical power system, Taiwan Power System (TaiPower System), with satisfactory results, and a comparison between HSAGA, simulated annealing, genetic algorithms and the hybrid partial gradient descent/simulated annealing method was also presented. (C) 1998 Elsevier Science Ltd. All rights reserved.
\end{abstract}

Keywords: VAR; Simulated annealing; Genetic algorithm

\section{Introduction}

The objective of VAR planning is to determine the minimum cost expansion pattern, in terms of locations, types and sizes, of new reactive power sources to be installed in power systems so as to ensure secure and economic operation. The dispatch of reactive powers can be effectively used to maintain acceptable voltage levels throughout the system and to reduce overall real power loss in the system. Furthermore, the security margin of power systems can be enlarged to reduce the possibility of voltage collapse by providing enough reactive power [1].

Because of its goal, VAR planning has commonly been formulated as a complicated constrained optimization problem with partially discrete, partially continuous and non-differentiable nonlinear objective functions [2-9]. A survey of literature on the problem reveals that various numerical optimization techniques have been employed to approach the complicated VAR planning problem. More specifically, Opoku [4] has formulated the problem as a mathematical optimization problem based on a linearized model (a restructured sparse admittance matrix) to reduce the dimensionality and computing time. Lebow [2], Granville [3] and Hong [5] and their colleagues have formulated the problem as a mixed-integer nonlinear programming problem with $0-1$ integer variables representing whether new reactive devices should or should not be installed. In

\footnotetext{
* Corresponding author.
}

this formulation, however, both the number and the value of capacitors were still treated as continuously differentiable variables. The generalized benders decomposition (GBD) technique [10] was then employed to decompose the problem into a continuous subproblem and an integer subproblem.

It should be noted that the above-mentioned methods can be classified as a greedy search technique. One main disadvantage of these techniques is that they often get stuck at a local optimum rather than at a global optimum. In order to circumvent this problem, Hsiao et al. [6, 7] applied the simulated annealing (SA) method to optimal VAR source planning in large-scale power systems. SA is a powerful, general-purpose stochastic optimization technique which can theoretically converge asymptotically to the global optimum solution with probability ' 1 '. One main drawback, however, of SA is that it takes much CPU time to find the global optimum.

Genetic algorithms (GA) were pioneered by Holland [11] and have proved useful and fast in a variety of search and optimization problems over the years. In power systems, Bakirtzis [12], Walters [13] and Wong [14] and their colleagues applied GA to the economic dispatch problem. Boone and Chiang [16] and Sundhararajan and Pahwa [17] used GA to solve optimal capacitor placement in distribution systems. Lee et al. [9] applied GA to optimal VAR planning. In the above applications, it is obvious that GA is popular for power system optimization problems.

In this paper, we present a hybrid simulated annealing/ 
genetic algorithm (HSAGA) method to reduce the CPU time of SA while retaining its main characteristics, i.e., the ability to get the near-global optimal solution in a finite time. A formulation of the optimal VAR planning as a constrained optimization problem is presented in Section 2. Then a detailed HSAGA algorithm is described in Section 3. In Section 4, the proposed algorithm is implemented in a software package and tested on a practical power system, TaiPower System, with promising simulation results. Also in Section 4, a comparison between HSAGA, SA, GA and hybrid partial gradient descent/simulated annealing [18] (HPGDSA) methods is presented. Finally, a summary and conclusion are given in Section 5.

\section{Problem formulation}

In this section, the VAR planning problem is formulated as a constrained optimization problem. First, we consider the objective function. This is expressed as the sum of the cost of VAR source placement and the cost of real power loss. Mathematically, the function is as follows:

$f\left(q_{c k}, q_{r k}, r_{k}\right)=C\left(q_{c k}, q_{r k}, r_{k}\right)+K_{e} D_{u} P_{\text {loss }}(\mathbf{x})$,

where $C\left(q_{c k}, q_{r k}, r_{k}\right)$ is the total cost of the VAR sources, and

$C=\sum_{k \in \Omega}\left(d_{k}+s_{c k} q_{c k}+s_{r k} q_{r k}\right) r_{k}$,

$\Omega$ is a set of all candidate buses to install VAR sources, $d_{k}$ is instalment cost at bus $k, s_{c k}$ is unit costs of capacitors, $S_{r k}$ is unit costs of reactors, $q_{c k}$ is added capacitive compensation at bus $k, q_{r k}$ is added inductive compensation at bus $k$, where $r_{k}$ is 0 for no placement of reactive power source at bus $k, 1$ for placement of reactive power source at bus $k, P_{\text {loss }}(\mathbf{x})$ is the real power loss in the system, $\mathbf{x}$ is the state vector of the system, $k_{e}$ is the energy cost per unit $(\$ / \mathrm{kW} \mathrm{h})$, and $D_{u}$ is the duration of the system operating time. Note that the function $f\left(q_{c k}, q_{r k}, r_{k}\right)$ is a mixed-integer nonlinear function. The independent variables are $q_{c k}, q_{r k}$ and $r_{k}$. The state vector $\mathbf{x}$ is a function of $q_{c k}, q_{r k}$ and $r_{k}$.

Next, we consider the constraint equations. Basically, there are two classes of constraint equations for our formulation. The first class consists of equality constraints stemming from conservation of power in an overall system. Mathematically, the equality constraint is expressed as follows:

$\mathbf{L}(\mathbf{x})=0$

where $\mathbf{L}(\mathbf{0})$ is a vector of power flow equations, and the argument, $\mathbf{x}$, is a state vector of the system, including the bus voltage magnitudes and angles. The other class comprises inequality constraints resulting from consideration of the following system security operation:

1. line flow limits,

2. voltage magnitude and phase angle difference limits,
3. voltage deviations,

4. transformer tap changing limits,

5. real and reactive power generation limits, and

6 . reactive power compensation limits.

Mathematically, these inequality constraints can be expressed in terms of a single inequality vector as follows:

$\mathbf{G}(\mathbf{x}) \leq 0$

Note that the inequality vector means that every component of the vector is an inequality equation.

In summary, combining Eqs. (1-3) gives us a complete formulation of the VAR planning problem as follows:

Minimize $\quad f\left(q_{c k}, q_{r k}, r_{k}\right)$

subject to $\quad \mathbf{L}(\mathbf{x})=0, \quad \mathbf{G}(\mathbf{x}) \leq 0$.

Remark 1: It is well recognized that formulating VAR planning as a constrained optimization problem has the advantage that optimization techniques can be systematically applied to the problem instead of trial-and-error exhaustive planning.

Remark 2: The constrained optimization problem is a complicated mixed-integer nonlinear problem. Previous experience shows that there are several local optima instead of a single one.

Remark 3: The complexity of the problem prevents the direct use of optimization techniques, for example linear programming, simulated annealing, gradient descent etc., without exploiting the nature of VAR planning.

\section{Hybrid simulated annealing/genetic algorithm approach}

We present, in this section, a hybrid stochastic optimization approach, namely the hybrid simulated annealing/ genetic algorithm (HSAGA), for solving the constrained optimization problem, Eq. (4). The HSAGA approach consists of a two-stage cycle. The first stage is the search by simulated annealing and the second stage is the evolution by the genetic operators. The basic configuration of HSAGA is shown in Fig. 1.

The first stage starts with a set of arbitrary strings (every string corresponds to some power network configuration with VAR source placement) from the population of current generation and at a certain temperature. Therefore, the approach can create a number of different search paths trying to find better solutions simultaneously. The SA method is utilized to guide these search paths when they climb in the search space. At a certain temperature, the strings visited by these search paths are joined to a set that is called a quasi-population. The completion of the first stage creates the quasi-population for the second stage. The genetic operation stage includes reproduction, crossover and mutation operators. Applying the genetic operators to the quasi-population can create the population 


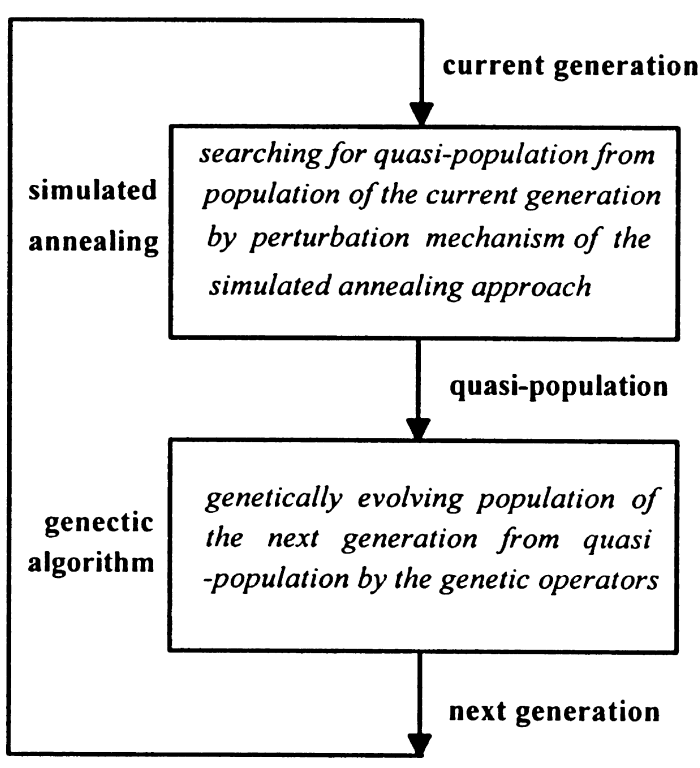

Fig. 1. The basic configuration of the HSAGA approach.

of the next generation. The cycle repeats until the stopping criteria are satisfied. In our approach, the high-fitness strings become the building blocks for the structures of future generations and are propagated in parallel, generation after generation. Therefore, the HSAGA can obtain a near-global optimal solution with speed in mind.

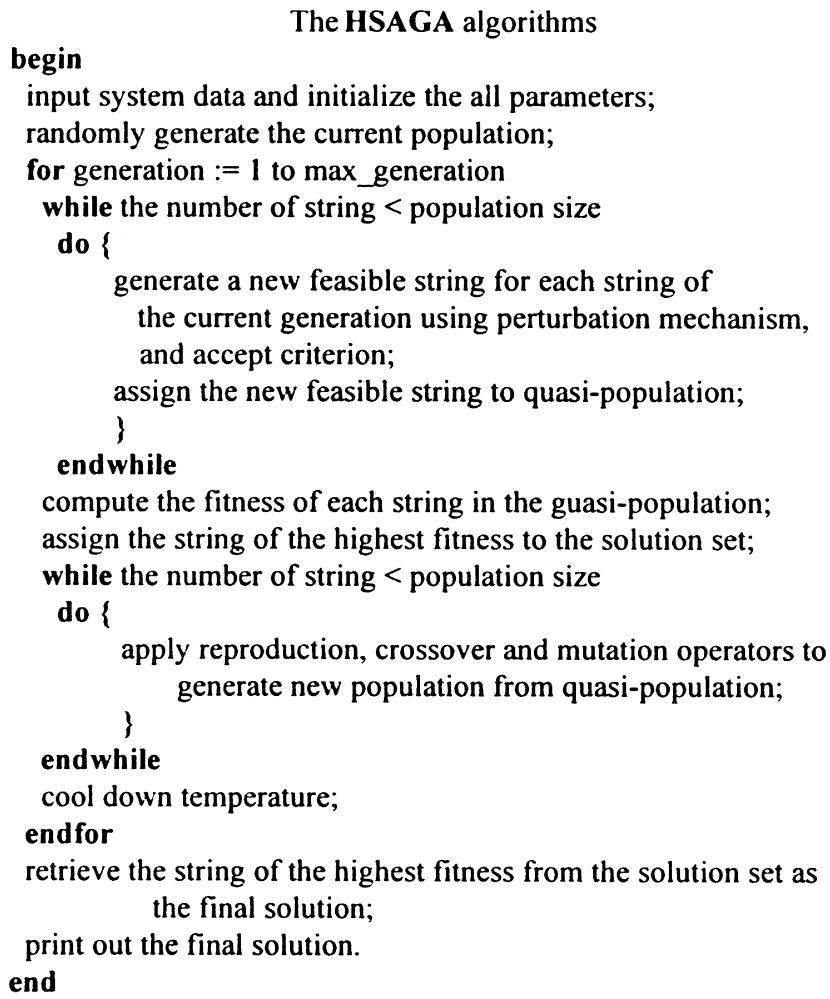

Fig. 2. Algorithm of the HSAGA. (a)

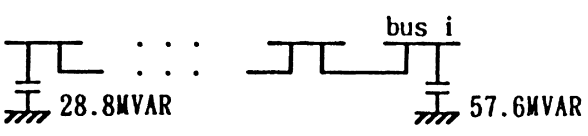

(b)

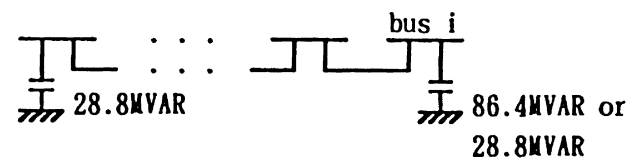

Fig. 3. The add/subtract move: (a) current configuration: (b) new configuration after the move.

The HSAGA algorithm is outlined in Fig. 2. Some explanations regarding the HSAGA are given in the following.

Explanation 1: The perturbation mechanism of HSAGA has four types of moves. These are described as follows:

1. add/subtract move:to add or subtract a preset realistic unit size, 28.8 MVAR, of reactive power source into a bus which was chosen from the candidate bus set by using a random number generator. The action of addition or subtraction is also determined by a random number (see Fig. 3);

2. multiplicative move:to add or subtract a reactive power source which is a positive integral multiple of a unit size of the reactive power source into a bus which was chosen from the candidate bus set by using a random number generator. The action of addition or subtraction is also determined by a random number (see Fig. 4);

3. interchange move:to interchange the reactive power sources of the two different buses on the basis of a random number generator (see Fig. 5);

(a)

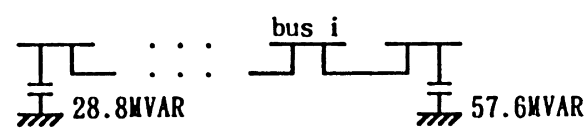

(b)

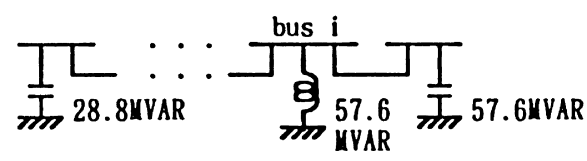

Fig. 4. The multiplicative move: (a) current configuration; (b) new configuration after the move.

(a)

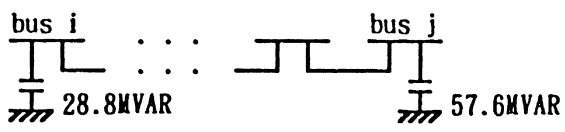

(b)

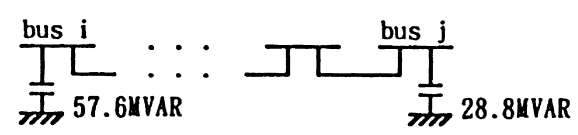

Fig. 5. The interchange move: (a) current configuration: (b) new configuration after the move. 


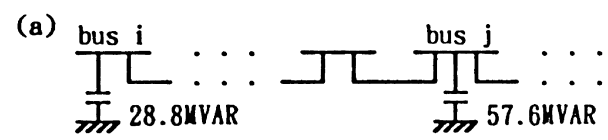

(b)

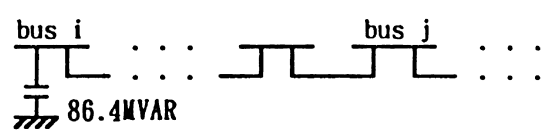

Fig. 6. The combinative move: (a) current configuration; (b) new configuration after the move.

4. combinative move:to move reactive power sources of one bus into the other bus, the selection being based on a random number generator (see Fig. 6).

Explanation 2: The genetic algorithm (GA) is a search mechanism based on the principle of natural selection and population genetics. The required design variables are encoded into a finite string corresponding to chromosomes in biological systems. Therefore, every string corresponds to some power network configuration with VAR source placement. Usually, the value of an element in the string is represented by a binary bit string [13, 15-17, 20]. For the VAR planning problem, a candidate solution corresponds to the locations, types and size for installing VAR sources in the candidate buses. It is difficult to use binary bit string as the candidate solution. Therefore, this paper proposes the use of integer number string to represent the candidate solution, as shown in Fig. 7. The integer string in Fig. 7 is decoded as: two banks of capacitors are located in the $(i-1)$ th candidate bus, no VAR sources are located in the $i$ th candidate bus, and one bank of reactors is located in the $(i+1)$ th candidate bus; and so on. The length of the string is equal to the number of total candidate buses. This representation method is easily implemented and can avoid the need to convert binary numbers into their decimal equivalents.

Explanation 3: The fitness function is a mapping to determine the fitness of each string in the population. The GA proceeds in the direction of evolving better-fit strings and the fitness value is the only information available to the GA. In other words, the performance of the algorithm is highly sensitive to the fitness values. In this paper, the fitness value is normalized between 0 and 1 by membership function;

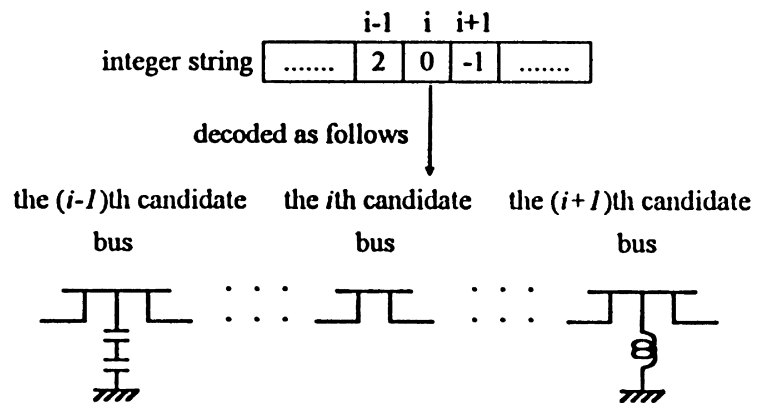

Fig. 7. Representation of integer string and its decoded network configuration. refer to Ref. [19] for details. The strings with larger fitness values are a better solution to the problem and have a higher probability of being selected. GA is basically an unconstrained search procedure in the original problem domain. To incorporate the constraints into the objective function, any string which violates the constraints is penalized by setting the fitness value to be zero. In this manner, GA implicitly becomes a constrained search procedure in the HSAGA.

Explanation 4: The reproduction operator is a probabilistic selection process in which strings are selected to produce offspring based on their fitness values. A simple method for this is called 'roulette wheel selection'; refer to Ref. [20] for details. This ensures that the expected number of a string is chosen in proportion to the string's fitness relative to the rest of the population. Strings with higher fitness values have a higher probability of contributing offspring and are simply copied into the next generation. In this paper, the string with the highest fitness in the population is directly copied into the next generation.

Explanation 5: The purpose of the crossover operator is to generate offspring strings from two parent strings. A typical method of crossover is the single-point crossover [16, 17]. This crossover chooses a random position in the string and swaps the elements either left or right of this point with another similarly partitioned string. This random position is called the crossover point. The performance of the single-point crossover can be improved by crossing over the strings at two points [14]. In the 2-point crossover, the crossover points are randomly determined. The elements between two crossover points are swapped between two parents to form two offspring. In this paper, the 2-point crossover operator is adopted.

Explanation 6: The mutation operator is a means of avoiding the loss of important information at a particular position in the string. The mutation operator with small mutation probability is applied to all the elements in each offspring string. The element is added or subtracted by one when mutation occurs, as shown in Fig. 8.

\section{Simulation results}

In this section, a practical power system, Taipower System, was tested by using the HSAGA. The system has 358 buses with 439 transmission lines planned in the future. A total of 56 buses is selected as candidate buses for installing new VAR sources. The candidate buses include weaker buses and more heavily loaded buses. In this test system, the

offspring \begin{tabular}{|c|c|c|c|c|c|c|c|}
\hline$\ldots \ldots$. & 2 & 1 & 0 & 2 & -1 & 4 & $\ldots \ldots$. \\
\hline
\end{tabular}
offspring mutation \begin{tabular}{|c|c|c|c|c|c|c|c|}
\hline$\ldots \ldots$. & 3 & 1 & 0 & 2 & -1 & 3 & $\ldots \ldots$. \\
\hline
\end{tabular}

Fig. 8. The mutation operator. 
Table 1

Results of HSAGA before and after planning for Taipower System

\begin{tabular}{lll}
\hline & Before planning & After planning \\
\hline Power loss (MW) & 302.0719 & 247.14 \\
Total cost $^{\mathrm{a}}$ & 6615.364258 & 5826.473633 \\
Total locations/total size (bank) & $18.18 \%$ & $11.93 \%$ \\
CPU time (min:s) & $42 / 114$ & \\
\hline
\end{tabular}

${ }^{\mathrm{a}}$ Million dollars (NT\$).

${ }^{\mathrm{b}}$ One bank $=28.8 \mathrm{MVAR}$.

following parameters are used: power loss per unit $K_{e}=$ NT $\$ 2.5 / \mathrm{kW} \mathrm{h}$, duration of the system operating time $D_{u}=$ 10 years, and are all limited to 20 banks. One bank of the VAR source is set at 28.8 MVAR, and the VAR source costs per unit are: $S_{c i}=s_{r i}=\mathrm{NT} \$ 3278880$ per bank and $d_{i}=$ NT\$520 000 per location. In the HSAGA approach, the control parameters are decided in many numerical tests. These parameters are set as follows: maximum generation is 30 , population size is 20 , crossover probability is 1 , mutation probability is 0.005 , initial temperature is 5 degrees, cooling rate is 0.95 and moves number is 7 .

A typical result of using the HSAGA technique is shown in Table 1, including the reduction power loss, cost, VAR source locations with size and the CPU time on a SUN SPARC 20 workstation in the computer center of National Taiwan University. A comparison between HSAGA, SA, GA and HPGDSA is shown in Table 2.

From these results, we make the following observations:

1. The HSAGA method can deal with a mixture of continuous and discrete variables. Moreover, it can be applied to a practical power system, Taipower System, with satisfactory results (see Table 1).

2. The solution obtained by HSAGA is a feasible solution. Because the fitness value of a non-feasible solution is set to be zero, the probability of a non-feasible solution being chosen is zero.

3. The solution time of HSAGA is much less than that of the conventional SA method and the solution quality is also improved slightly. Moreover, comparing HSAGA, SA, GA and HPGDSA, the solution quality of HSAGA is the best. However, the solution speed of HPGDSA is the fastest.

Table 2

Comparison between HSAGA, SA, GA and HPGDSA for Taipower System

\begin{tabular}{llll}
\hline Method & Total cost ${ }^{\mathrm{a}}$ & $\begin{array}{l}\text { Total locations/ } \\
\text { total size (bank) }\end{array}$ & $\begin{array}{l}\text { CPU time } \\
\text { (min:s) }\end{array}$ \\
\hline HSAGA & 5826.473633 & $42 / 114$ & $20: 28$ \\
SA & 5853.925781 & $31 / 94$ & $136: 23$ \\
GA & 5865.719238 & $46 / 125$ & $4: 14$ \\
HPGDSA & 5834.754883 & $48 / 126$ & $1: 51$ \\
\hline
\end{tabular}

${ }^{\mathrm{a}}$ Million dollars (NT\$).

${ }^{\mathrm{b}}$ One bank $=28.8$ MVAR.
4. All parameters of the test system used in the algorithm are industrial specifications. Therefore, the solutions of the HSAGA algorithm are ready for industrial use.

5. In the above testing system, we can obtain very close results in many numerical tests. Therefore, these results are near-global optimal solutions.

\section{Conclusions}

In this paper, a relatively realistic problem formulation for the optimal VAR source planning problem is presented. The problem formulation is treated as a constrained and non-differentiable optimization problem. The formulation includes the cost of system operation, investment cost of VAR sources, load and operational constraints. They are the factors of most concern in the VAR planning problem.

We have developed a fast near-global optimization approach, the hybrid simulated annealing/genetic algorithm, for solving the VAR planning problem in large-scale electric power systems. The basic configuration of HSAGA consists of a two-stage cycle. The first stage is the search by simulated annealing and the second stage is the evolution by genetic operators. The solution algorithm is efficiently applied to a practical power system, Taipower System, with satisfactory results. The prominent feature of the HSAGA is that its solution time is much less than that of the conventional simulated annealing method and the solution quality is also improved slightly. Therefore, the HSAGA algorithm has the potential to be a practical near-global optimal solution to the VAR planning problem in large-scale electric power systems.

\section{References}

[1] Chen YL, Liu CC. Optimal multi-objective VAR planning using an interactive satisfying method. IEEE Transactions on Power Systems 1995;10(2):664-670.

[2] Lebow WM, Rouhani R, Nadira R, Usoro PB, Mehra RK, Sobieski DW, Pal MK, Bhavaraju MP. A hierarchical approach to VAR optimization in system planning. IEEE Transactions on PAS 1985;104(8):2051-2057.

[3] Granville S, Pereira MVF, Monticelli A. An integrated methodology for VAR sources planning. IEEE Transactions on Power Systems 1988;3(2):549-557. 
[4] Opoku G. Optimal power system VAR planning. IEEE Transactions on Power Systems 1990;5(1):53-59.

[5] Hong YY, Sun DI, Lin SY, Lin CJ. Multi-year multi-case optimal VAR planning. IEEE Transactions on Power Systems 1990;5(4):1294-1301.

[6] Hsiao YT, Liu CC, Chiang HD, Chen YL. A new approach for optimal VAR sources planning in large scale electric power systems. IEEE Transactions on Power Systems 1993;8(3):988-996.

[7] Hsiao YT, Chang HD, Liu CC, Chen YL. A computer package for optimal multi-objective VAR planning in large scale power systems. IEEE Transactions on Power Systems 1994;9(2):668-676.

[8] Lee KY, Ortiz JL, Park YM, Pond LG. An optimization technique for reactive power planning of subtransmission network under normal operation. IEEE Transactions on Power Systems 1986;1(2):153-159.

[9] Lee KY, Bai X, Park YM. Optimization method for reactive power planning by using a modified simple genetic algorithm. IEEE Transactions on Power Systems 1995;10(4):1843-1850.

[10] Geoffrion AM. Generalized benders decomposition. JOTA 1972;10(4):237-261.

[11] Holland JH. Adaptive behavior in natural and artificial systems. Ann Arbor, MI: University of Michigan Press, 1975.

[12] Bakirtzis A, Petridis V, Kazarlis S. Genetic algorithm solution to the economic dispatch problem. IEE Proceeding-C 1994;141(4):377382.
[13] Walters DC, Sheble GB. Genetic algorithm solution of economic dispatch with valve point loading. IEEE Transactions on Power Systems 1993;8(3):1325-1332.

[14] Wong KP, Wong YW. Genetic and genetic/simulated-annealing approaches to economic dispatch. IEE Proceeding-C 1994;141(5):507-513.

[15] Huang CL, Tzeng JS, Yang PC, Yang HT. Implementation of genetic algorithm for unit commitment. 14th Symposium on Electrical Power Engineering, Taiwan, December 1993:439-446.

[16] Boone G, Chiang HD. Optimal capacitor placement in distribution systems by genetic algorithm. Electrical Power and Energy Systems 1993;15(3):155-162.

[17] Sundhararajan S, Pahwa A. Optimal selection of capacitors for radial distribution systems using a genetic algorithm. IEEE Transactions on Power Systems 1994;9(3):1499-1507.

[18] Liu CW, Jwo WS, Liu CC, Hsiao YT. A fast global optimization approach to VAR planning for the large scale electric power systems. IEEE Transactions on Power Systems 1997;12(1):437-443.

[19] Jwo WS, Liu CW, Liu CC, Hsiao YT. Hybrid expert system and simulated annealing approach to optimal reactive power planning. IEE Proceeding-C 1995;142(4):381-385.

[20] Goldberg DE. Genetic algorithms in search, optimization and machine learning. Reading, MA: Addison-Wesley, 1989. 